\title{
Élevage artificiel des agneaux
}

630.4

$\mathrm{C} 212$

P 1507

1990

$\mathrm{fr}$. $00 \mathrm{Ag}$

c. 3

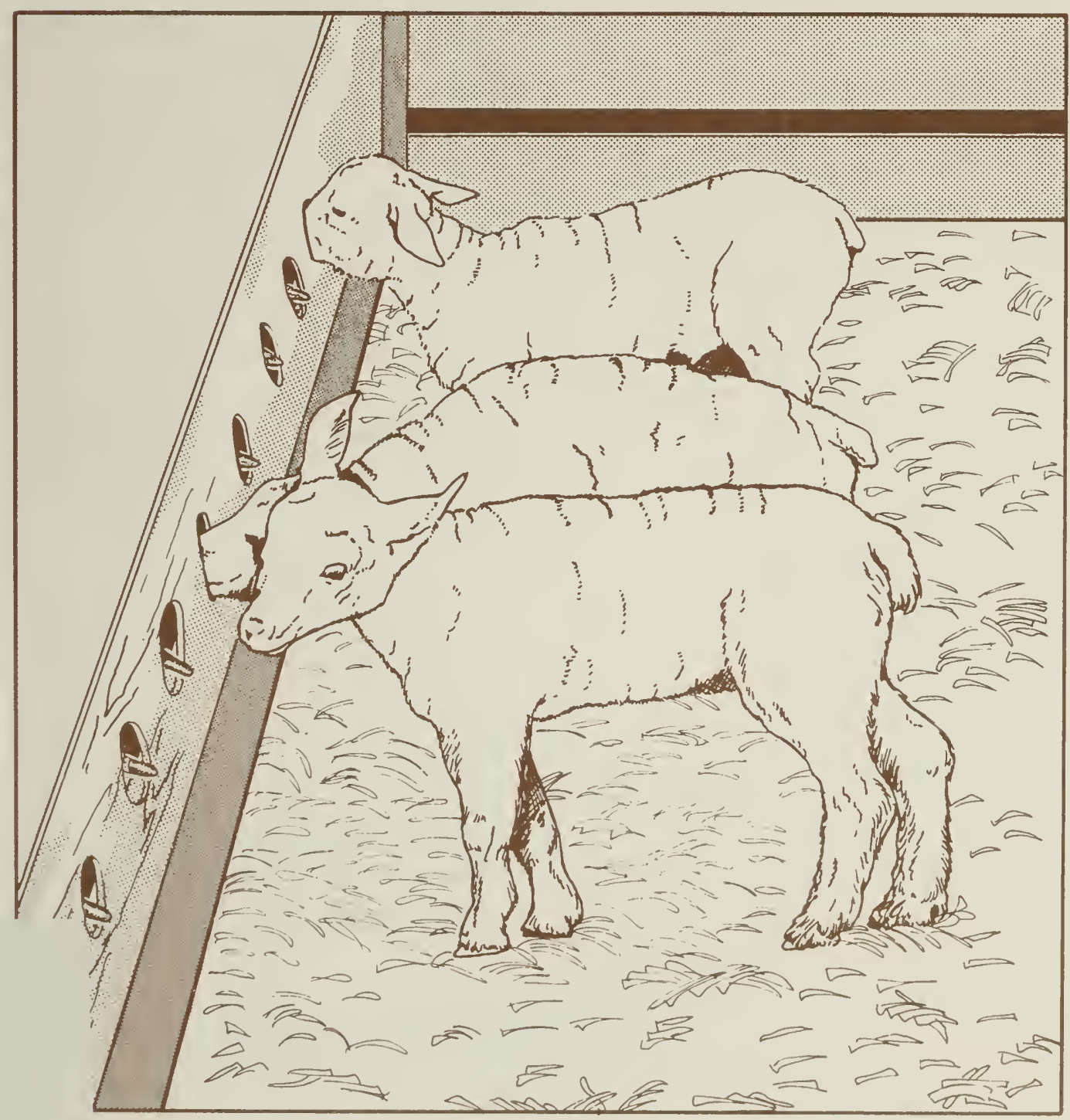


Digitized by the Internet Archive in 2012 with funding from

Agriculture and Agri-Food Canada - Agriculture et Agroalimentaire Canada 


\section{Élevage artificiel des agneaux}

Version originale rédigée par

A.D.L. Gorrill, G.J. Brisson, D.B. Emmons, et G.J. St-Laurent

Première révision par

J.W.G. Nicholson

Deuxième révision par

D.P. Heaney et D.A. Leger

Centre de recherches zootechniques

Ottawa (Ont.)

Agriculture Canada Publication 1507/F

On peut en obtenir des exemplaires à la

Direction générale des communications,

Agriculture Canada, Ottawa K1A 0C7

- Ministre des Approvisionnements et Services Canada 1990

No de cat. A63-1507/1990F ISBN 0-662-95875-6

Impression 1972 Révision 1978 Révision 1982 Réimpression 1985

Réimpression 1989 Révision 1990 2,5M-6:90

Also available in English under the title

Artificial rearing of young lambs 


\section{PRÉFACE}

Depuis sa première parution en 1972 , la présente publication a été révisée par les auteurs originaux en 1978 et a fait l'objet d'une révision en profondeur par J.W.G. Nicholson en 1982. Nous tenons à remercier les auteurs des éditions précédentes qui ont grandement contribué au progrès du secteur des ovins au Canada.

La présente révision a été préparée dans le but de mettre à jour le guide d'élevage artificiel des agneaux, de façon à répondre à l'intérêt manifeste et soutenu que connaît ce domaine. Elle puise abondamment à même les résultats de recherches réalisées aux EtatsUnis et au Canada. Cette publication a également tiré profit des travaux de recherches et de la vaste expérience du Centre de recherches zootechniques d'Ottawa, où plus de 50000 agneaux ont été élevés artificiellement entre 1970 et 1989. 


\section{TABLE DES MATIÈRES}

Préface 4

Points saillants 6

Introduction 8

Choix et préparation des agneaux 8

Réduction au minimum de l'élevage artificiel 8

Alimentation au colostrum 9

Séparation d'avec la brebis 10

Apprentissage de l'allaitement artificiel 11

Formation de groupes d'agneaux selon la taille et l'âge 12

Conduite de l'élevage artificiel 13

Techniques d'allaitement 13

Eau et alimentation complémentaire 13

Température de l'aliment d'allaitement 15

Sevrage précoce 16

Choix de l'aliment d'allaitement 17

Sortes 17

Préparation 19

Addition de formaline 19

Systèmes de distribution 20

Protection des tétines 20

Alimentation au seau à tétines 20

Nourrisseurs à plusieurs tétines 21

Rampes nourricières 22

Hygiène 28

Maladies et troubles digestifs 28

Performance des agneaux nourris aux aliments d'allaitement 29

Lectures conseillées 30 


\section{POINTS SAILLANTS}

On dispose aujourd'hui de préparations d'aliments d'allaitement, de systèmes d'alimentation et de conduite de l'élevage artificiel qui permettent d'élever avec succès de très jeunes agneaux. En effet, il existe une technologie qui permet d'élever artificiellement, et de façon économique, les agneaux surnuméraires de races prolifiques qui produisent plus d'agneaux que les brebis ne peuvent en nourrir, de même que les agneaux qui sont devenus orphelins à la suite d'une production insuffisante de lait maternel, de la mort de la brebis ou du rejet par celle-ci. L'élevage artificiel n'est cependant pas recommandé pour tous les agneaux de la saison.

Le succès de l'élevage artificiel des agneaux nourris avec des aliments d'allaitement repose sur le respect des règles suivantes :

- S'assurer que les agneaux reçoivent du colostrum dès leur naissance, soit par allaitement naturel durant au moins $6 \mathrm{~h}$, ou par administration au biberon à raison de $50 \mathrm{~mL}$ par kilogramme de poids corporel.

- Pour faciliter l'entraînement, séparer les agneaux de la brebis aussitôt que possible après qu'ils ont reçu le colostrum.

- S'assurer que les agneaux ont appris à boire aux tétines artificielles, mais ne pas prolonger l'apprentissage inutilement.

- Pour obtenir de meilleurs résultats, utiliser des aliments d'allaitement en poudre dosant environ $24 \%$ de matières grasses et $24 \%$ de protéines.

- Préparer l'aliment d'allaitement de façon à ce qu'il contienne de 16 à $20 \%$ de matières solides.

- Ajouter de 0,05 à $0,10 \%$ de formaline à l'aliment liquide afin d'empêcher la croissance des bactéries, d'éviter que le lait ne surisse et de réduire les risques de météorisme de la caillette (lire auparavant la section «Addition de formaline»).

- La rampe nourricière constitue une méthode simple, peu coûteuse et efficace pour nourrir des groupes d'agneaux. Il faut toutefois placer des protecteurs autour des tétines pour empêcher les agneaux de les mâchonner.

- Choisir les agneaux qui ont à peu près le même âge et le même poids pour former des groupes de 12 ou moins. La plupart des agneaux s'adaptent vite au système d'alimentation et apprennent à sucer les tétines au bout d'un ou deux jours.

- Servir l'aliment d'allaitement à volonté. Les agneaux auront une croissance au moins égale à celle des agneaux allaités par la brebis.

- Nettoyer régulièrement le système de distribution et les ustensiles, et toujours employer des méthodes hygiéniques.

- Fournir en tout temps de l'eau fraîche, des aliments complémentaires appétents et nutritifs contenant de 17 à $20 \%$ de protéines, et du foin de luzerne de haute qualité. 
- Administrer aux agneaux des injections de vitamines A, D et E, ainsi que de sélénium si la dystrophie musculaire sévit dans la région.

- Vacciner les agneaux contre l'entérotoxémie (rein pulpeux) avec une préparation multiple de clostridium.

Surtout ne pas oubliez que la conduite est le facteur déterminant du succès. 


\section{INTRODUCTION}

L'allaitement artificiel consiste à séparer très tôt les agneaux de leur mère et à les nourrir aux aliments d'allaitement. Cette pratique est de plus en plus fréquente, probablement à cause de l'utilisation de races prolifiques, qui produisent plus d'agneaux que les brebis ne peuvent en nourrir. Les méthodes recommandées d'élevage artificiel sont des moyens à la fois utiles et profitables pour élever ces agneaux surnuméraires, de même que les agneaux qui sont devenus orphelins à cause d'insuffisance de lait maternel, de la mort de la brebis ou d'un rejet par celle-ci.

Les agneaux orphelins sont depuis longtemps élevés artificiellement. On utilisait parfois du lait de vache entier, mais plus fréquemment du lait écrémé, souvent avec des résultats insatisfaisants compte tenu des frais et des efforts engagés. C'est en Europe où existaient de vastes secteurs d'élevage de brebis laitières destinées à la production du fromage qu'ont été effectuées une bonne partie des premières recherches pour mettre au point des aliments d'allaitement efficaces et des systèmes de conduite de l'élevage artificiel des agneaux.

Dans les premiers débuts de l'élaboration d'aliments d'allaitement synthétiques, les chercheurs ont constaté que l'administration de lait de vache entier, séché par pulvérisation, entraînait des gains de poids comparables à ceux obtenus en élevage naturel ou en élevage artificiel au lait de brebis frais ou lait congelé reconstitué. Cependant, dans les conditions normales, le coût d'utilisation de lait de vache entier reconstitué, séché par pulvérisation, était prohibitif. C'est pourquoi on s'est orienté vers la mise au point d'aliments d'allaitement artificiels, à base de poudre de lait écrémé de pulvérisation et de mélanges de graisses animales et végétales. Ces aliments d'allaitement ont donné lieu à une croissance similaire à celle obtenue avec le lait de vache entier, reconstitué.

Des études subséquentes, réalisées en Europe et en Amérique du Nord, ont permis de perfectionner tant les préparations d'aliments d'allaitement que les systèmes de conduite de l'allaitement artificiel des agneaux. La présente publication fait la synthèse de ces travaux sous forme de lignes directrices pratiques qui permettent de mener à bien l'élevage artificiel.

\section{CHOIX ET PRÉPARATION DES AGNEAUX}

\section{REDUCTION AU MINIMUM DE L'ÉLEVAGE ARTIFICIEL}

Ne pas pratiquer l'allaitement artificiel s'il n'est absolument nécessaire. Dans les exploitations commerciales, l'élevage artificiel de tous les agneaux n'est pas recommandé. Il est plus économique de 
laisser les brebis élever elles-mêmes autant d'agneaux qu'elles peuvent en nourrir de façon adéquate et de ne recourir à l'allaitement artificiel que pour l'excédent. On conseille également autant que possible de faire adopter des agneaux surnuméraires par d'autres brebis. L'élevage artificiel peut cependant être plus avantageux lorsque l'on pratique la remise à la reproduction très précoce des brebis, étant donné que l'allaitement peut inhiber le cycle oestral. Dans ce cas, il faudrait que le supplément d'agneaux produits à la suite de la remise à la reproduction précoce devrait être suffisant pour compenser les coûts additionnels de l'allaitement artificiel. On devrait pouvoir obtenir facilement trois agnelages en 2 ans en sevrant les agneaux allaités naturellement à l'âge de 6 à 8 semaines.

Il est important de bien choisir, dans les grosses portées, les agneaux qu'on élèvera artificiellement. Auparavant, on conseillait de choisir les agneaux les plus gros, les plus vigoureux, croyant que les agneaux petits et faibles avaient de meilleures chances de survie s'ils étaient laissés avec la mère. Cette recommandation ne se justifie plus guère et cela pour deux raisons : premièrement, ce sont les agneaux les plus gros qui valorisent au mieux le lait de la brebis, parce qu'ils sont plus en mesure de tirer profit de ses capacités laitières; deuxièmement, de vastes recherches ont démontré que les agneaux les plus petits peuvent être élevés facilement de façon artificielle, souvent avec plus de succès que s'ils étaient élevés naturellement. Le meilleur compromis est de laisser les deux (ou trois si la brebis est une mère et une laitière exceptionnelle) agneaux les plus homogènes avec la brebis et d'élever artificiellement les autres.

\section{ALIMENTATION AU COLOSTRUM}

Les agneaux viennent au monde dépourvus d'anticorps dont le rôle est de protéger contre les maladies infectieuses courantes. Ces anticorps leur sont transmis dans le premier lait de la brebis, le colostrum, qu'ils boivent peu de temps après la naissance. En plus de fournir les anticorps essentiels, le colostrum est riche en substances nutritives comme les vitamines, les protéines et les matières grasses nécessaires au nouveau-né pour partir «sur un bon pied». En outre, le colostrum est un laxatif doux, qui contribue à excréter le méconium qui tapisse le système digestif à la naissance, et qui aide au bon fonctionnement de l'intestin.

Dans les premières $24 \mathrm{~h}$ après leur naissance, les agneaux peuvent absorber les anticorps intacts à partir de leur intestin. Cependant, ce mécanisme d'absorption commence à se résorber peu après la première tétée de l'agneau, de sorte que les anticorps sont alors digérés avant d'être absorbés. Il est donc important que le premier aliment que l'agneau reçoive soit du colostrum et non un aliment d'allaitement ou une solution sucrée.

Les agneaux destinés à l'élevage artificiel doivent pouvoir têter le colostrum de leur mère durant au moins $6 \mathrm{~h}$ avant d'être séparés de 
cette dernière. Si, pour quelque raison, les agneaux ne peuvent obtenir le colostrum de leur mère, on peut extraire à la main le colostrum en excès d'une autre brebis et le servir soit au biberon ou à l'aide d'un tube de gavage. Le colostrum congelé, réchauffé à la température du corps et servi au biberon, est également une bonne solution. Bien qu'il soit préférable d'utiliser du colostrum congelé de brebis, le colostrum de vache fournit également aux agneaux les anticorps nécessaires. On doit administrer au moins $50 \mathrm{~mL}$ de colostrum par kilogramme de poids de l'agneau pour lui conférer une résistance suffisante aux maladies. Lors de la décongélation du colostrum, on évitera de trop le chauffer, car une température supérieure à la température du corps $\left(40^{\circ} \mathrm{C}\right)$ détruit une partie des anticorps.

La concentration en anticorps du colostrum décroît à chaque tétée après la mise bas. Il faut donc recueillir et congeler le plus tôt possible le colostrum en excès afin d'obtenir un taux élevé d'anticorps.

\section{SÉPARATION D'AVEC LA BREBIS}

Les agneaux destinés à l'élevage artificiel peuvent être enlevés à leur mère dès qu'on estime qu'ils ont reçu une quantité appropriée de colostrum, c'est-à-dire au bout d'environ $6 \mathrm{~h}$. Cependant, comme on l'a déjà mentionné, si la brebis n'a pas assez de lait à ce moment-là pour nourrir tous ses agneaux, il faut leur donner une quantité supplémentaire de colostrum à l'aide d'un biberon ou d'un tube de gavage.

Plus tôt les agneaux sont séparés de leur mère, plus il sera facile de les entraîner. Après $48 \mathrm{~h}$, le lien qui se crée entre la mère et ses petits devient tellement fort que la séparation est traumatisante. En outre, après cette période, le réflexe de têtage est à ce point développé chez les agneaux qu'il est alors difficile de leur apprendre à boire à l'aide d'une tétine artificielle. Il est aussi plus difficile de tarir la brebis sans endommager le pis.

Aussitôt que les agneaux sont séparés de leur mère, on les placera dans un enclos chaud et sec, à l'abri des courants d'air et assez loin de la brebis pour qu'ils ne puissent l'entendre ni la voir. Il faut prévoir un espace minimal de $0,2 \mathrm{~m}^{2}$ par agneau. Les cases dotées d'un plancher de métal déployé ou grillagé sont souvent utilisées, mais on peut aussi employer des parquets au sol garnis d'une litière épaisse, à condition d'accorder plus d'espace à chaque agneau $\left(0,5 \mathrm{~m}^{2}\right)$. Afin de permettre une meilleure absorption des substances nutritives et un croît plus rapide, la température de la pièce doit être maintenue à près de $20^{\circ} \mathrm{C}$.

Une fois que les agneaux ont reçu le colostrum, on les fera jeûner quelques heures avant de les initier à la nourrice artificielle. Pour faciliter l'apprentissage, un jeûne d'environ $6 \mathrm{~h}$ est recommandé. Cependant, les petits agneaux ou les agneaux chétifs ne doivent pas jeûner plus de 2 à $4 \mathrm{~h}$. Lorsque les agneaux ont faim, la première goutte de l'aliment d'allaitement suffit d'habitude à déclencher la tétée. 


\section{APPRENTISSAGE DE L'ALLAITEMENT ARTIFICIEL}

Pour apprendre à l'agneau à boire, placer la tétine dans sa bouche et, avec la main, remuer sa mâchoire pour l'inciter à téter (fig. 1). Beaucoup de brebis poussent doucement leurs nouveau-nés sur la croupe pour les guider jusqu'au pis pour leur première tétée. Certains éleveurs croient qu'en imitant ce geste, c'est-à-dire en plaçant une main sur la croupe de l'agneau et en le poussant doucement, ils peuvent l'inciter à téter. Chez la plupart des agneaux, le goût du lait tiède qui provient de la nourrice artificielle suffit à déclencher le réflexe de succion. Une ou deux séances d'entraînement suffisent habituellement, mais, durant les premiers jours, il faut surveiller de près les agneaux logés en groupe pour s'assurer qu'ils boivent tous comme il faut. Une lampe placée au-dessus de l'aire d'alimentation attire les agneaux et facilite leur adaptation au nouveau système de distribution.

Durant la phase d'entraînement, l'aliment d'allaitement peut être servi chaud ou froid. Il est cependant avantageux de le servir toujours à la même température pendant toute la durée du programme. Les agneaux accepteront peut-être plus facilement l'aliment chaud durant la phase d'apprentissage, mais il peut survenir des problèmes de rejet si on le remplace plus tard par un aliment froid, ce qui nécessitera un autre entraînement. Pour les mêmes raisons, les tétines utilisées

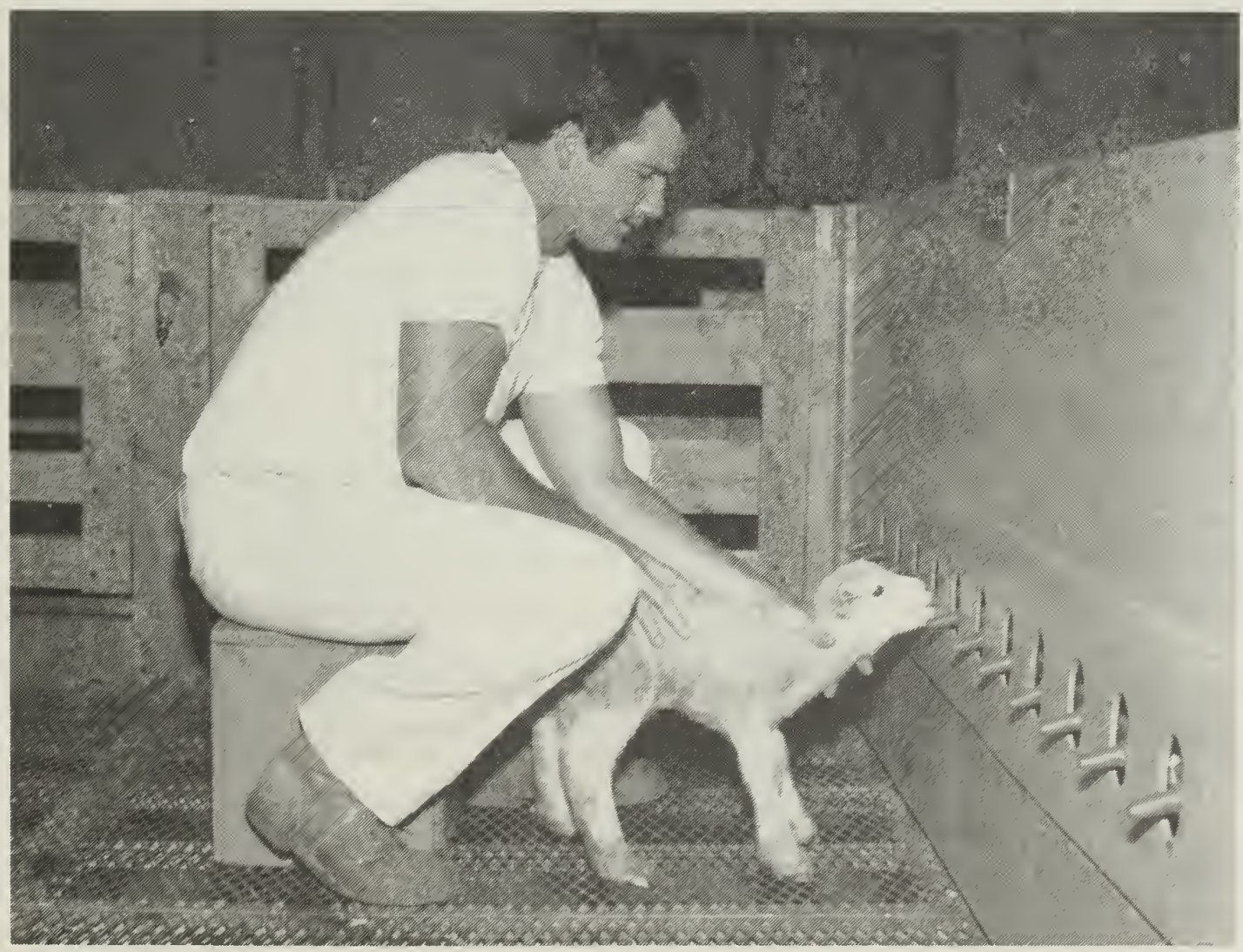

Fig. 1 Agneau apprenant à se nourrir à la rampe nourricière (d'après Ainsworth et al., 1987). 
durant l'apprentissage doivent être les mêmes qui seront utilisées plus tard.

Il faut éviter d'accorder trop d'attention aux agneaux nouveaunés, car un lien social risque de se créer entre les agneaux et leur gardien. Il est alors possible que l'agneau ne veuille ingérer l'aliment d'allaitement que si le gardien est présent ou se trouve à proximité, et qu'il soit désemparé s'il est laissé seul. Certes il est important de s'assurer que les nouveau-nés sont à l'aise et se nourrissent bien, mais il ne faut pas les rendre dépendants de la présence de leur gardien. Il peut être mignon de voir un petit agneau vous suivre partout, mais quand il s'agit de tout un troupeau, c'est une autre histoire!

La présence d'un ou de deux agneaux qui ont déjà appris à boire l'aliment d'allaitement sera utile pour apprendre aux autres agneaux. Il faut toutefois bien choisir ces agneaux bien dressés. S'ils sont beaucoup plus gros que les nouveaux agneaux, ces derniers peuvent les prendre pour une mère suppléante et essayer de leur téter le nombril ou le scrotum. Si cela se produit, le jeune agneau doit alors être séparé des autres et nourri à part jusqu'à ce qu'il apprenne à boire à la tétine artificielle.

Les agneaux qui ne se sont pas adaptés au système d'allaitement au bout de $48 \mathrm{~h}$ n'apprendront probablement jamais. Il faut alors les nourrir au biberon sinon ils mourront de faim.

L'expérience a démontré que certaines races apprennent plus facilement que d'autres à s'allaiter à la nourrice artificielle. C'est notamment le cas de la Dorset et de la Finnoise ainsi que des sujets croisés de ces deux races. Cependant, on a réussi à élever artificiellement des agneaux qui provenaient de plus de 30 races et croisements, ce qui démontre qu'avec les soins nécessaires, cette méthode peut convenir à la plupart, sinon à la totalité des races.

\section{FORMATION DE GROUPES D'AGNEAUX SELON LA TAILLE ET L'ÂGE}

La croissance des agneaux est supérieure quand ces derniers sont nourris par groupes de 12 ou moins, bien qu'un grand nombre d'exploitations aient eu de bons résultats avec des groupes de 25 à 50 agneaux. Le principal désavantage des grands groupes est l'incidence plus élevée de cas de tétée du nombril ou d'autres formes de pica. On évitera de placer les agneaux nouveau-nés avec des agneaux qui sont âgés de plus d'une semaine parce que le jeune peut adopter le plus vieux comme mère suppléante et essayer de le téter plutôt que de s'allaiter à la nourrice artificielle.

Il faut grouper les agneaux qui sont le plus homogènes possible, en ce qui concerne la taille et l'âge. Une fois qu'un groupe d'agneaux est formé, des liens sociaux s'établissent entre eux. Il vaut mieux, si possible, garder le groupe intact durant toute la période de croissance. La croissance des agneaux sera meilleure s'ils ne sont pas soumis au stress du départ d'anciens amis ou partenaires et de la création de 
nouveaux liens. Au moment du passage à l'alimentation solide, après le sevrage des aliments d'allaitement, il est habituellement plus efficace de former de plus grands groupes d'agneaux. Il est préférable, pour la préservation des liens sociaux, de joindre deux groupes entiers ou plus d'agneaux, plutôt que de prendre quelques agneaux de plusieurs petits groupes pour former un groupe plus grand.

\section{CONDUITE DE L'ÉLEVAGE ARTIFICIEL}

\section{TECHNIQUES D'ALLAITEMENT}

Le biberon peut être utilisé avec succès dans l'élevage artificiel. Si l'on choisit cette méthode, il faut nourrir les agneaux à satiété toutes les $6 \mathrm{~h}$ durant les 3 ou 4 premiers jours. On pourra ensuite les nourrir deux et préférablement trois fois par jour. L'allaitement au biberon permet de rationner la consommation à partir du moment où les agneaux ont 3 ou 4 jours. La méthode présente le double avantage de réduire les coûts des aliments d'allaitement et d'amoindrir le ralentissement de croissance au sevrage (voir la section "Sevrage précoce»). Cependant, si l'on a plusieurs agneaux à nourrir, les coûts en main-d'oeuvre deviennent vite prohibitifs. Il devient donc nécessaire, pour réduire les coûts, d'utiliser une technique d'allaitement à volonté, où l'aliment d'allaitement est disponible en tout temps, de façon à ce que les agneaux puissent satisfaire leur appétit en s'alimentant par eux-mêmes. Les théories et lignes directrices présentées dans cette brochure s'appliquent particulièrement à l'allaitement à volonté, bien que pour la plupart elles peuvent cependant s'adapter à l'allaitement au biberon.

Un grand nombre de systèmes (voir la section «Systèmes de distribution»), allant du seau à tétines au lactoduc perfectionné, en passant par la rampe nourricière, sont disponibles pour l'allaitement à volonté. Le choix du système dépend du nombre d'agneaux à nourrir ainsi que des circonstances ou de préférences individuelles. Peu importe le système adopté, chaque tétine peut nourrir au moins 4 ou 5 agneaux (fig. 2). En conditions expérimentales, on a pu élever jusqu'à 10 agneaux par tétine.

\section{EAU ET ALIMENTATION COMPLEMENTAIRE}

Il est très important que les agneaux aient à leur disposition de l'eau et un aliment complémentaire de qualité et appétent durant toute la période où ils sont au régime liquide. Bien que faible, la consommation d'aliments complémentaires permettra aux agneaux de s'habituer aux aliments solides et de développer leur rumen, deux conditions essentielles pour le passage harmonieux de l'alimentation liquide à l'alimentation solide. 


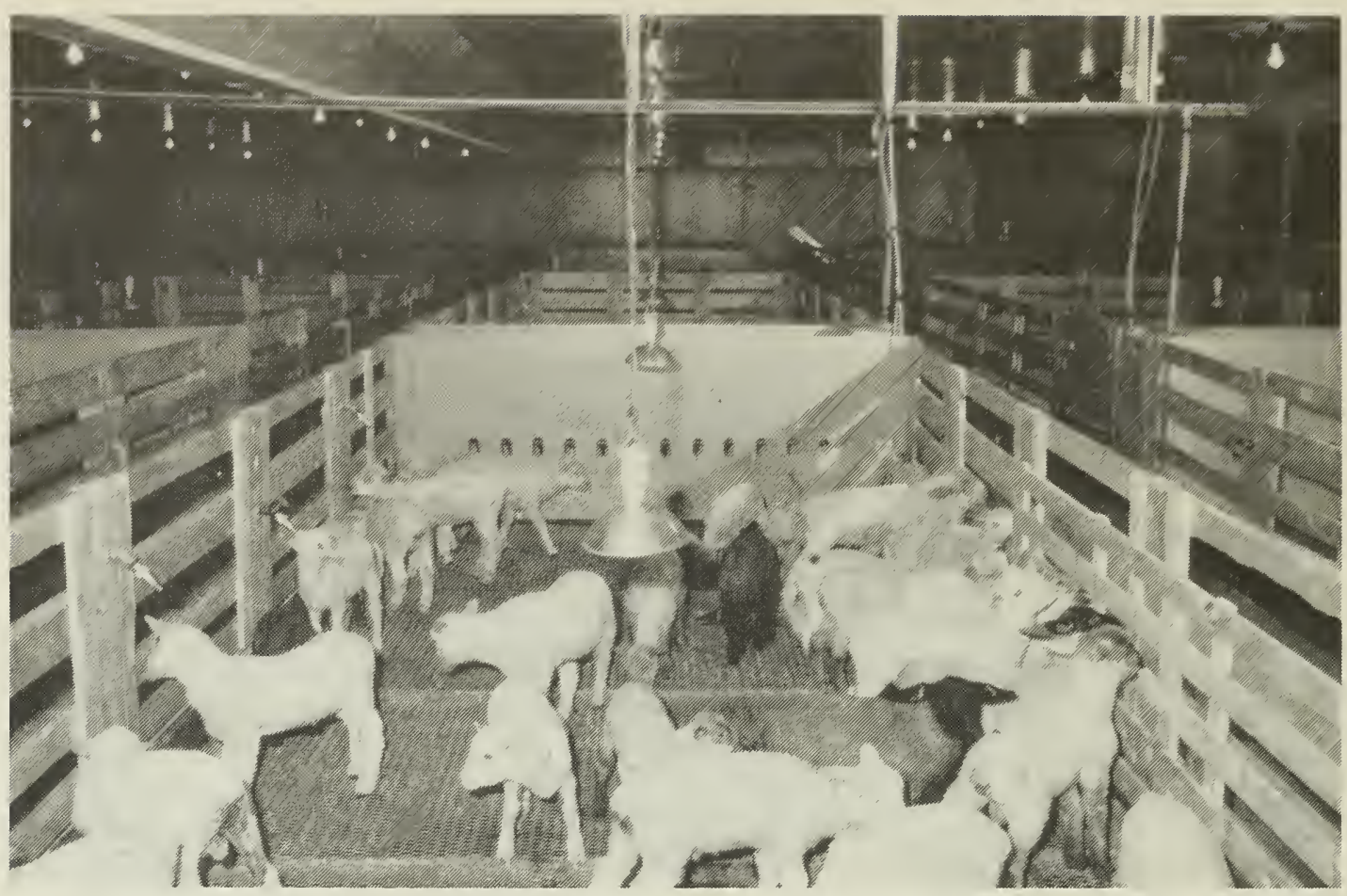

Fig. 2 Enclos pour agneaux nourris à l'aliment d'allaitement (d'après Ainsworth et al., 1987).

L'aliment concentré complémentaire doit contenir de 17 à $20 \%$ de protéines brutes. Il doit avoir une faible teneur en cellulose brute et une grande valeur énergétique. Le glucose et la mélasse sont de très bonnes sources d'énergie, en plus d'être fort appétentes. Le concentré peut être présenté en farine ou en agglomérés. La présentation sous forme d'agglomérés empêche la tendance à manger de façon sélective. Cependant, il est recommandé d'émietter les agglomérés, car les très jeunes agneaux refusent parfois de les manger lorsqu'ils sont durs. En plus du concentré, on offrira aux agneaux un bon foin de luzerne à tiges fines.

Les aliments commerciaux de démarrage et de croissance pour veaux, les aliments de démarrage pour porcs (pourvu qu'ils ne contiennent pas de cuivre), ainsi que les aliments de démarrage pour agneaux à forte teneur en protéines brutes ont donné des résultats satisfaisants comme aliments complémentaires.

Il faut s'assurer que l'aliment complémentaire demeure frais et éviter qu'il ne soit souillé par du fumier ou des moisissures. Enlever la nourriture non consommée et remettre de l'aliment frais tous les jours. Il vaut mieux disposer les mangeoires de façon à ce que les agneaux ne puissent pas monter dessus. On peut les suspendre à l'extérieur de l'enclos et ménager une ouverture pour la tête de l'agneau. L'ouverture doit mesurer de 12 à $15 \mathrm{~cm}$ de hauteur et être située de 30 à $35 \mathrm{~cm}$ au-dessus du sol de l'enclos. 


\section{TEMPÉRATURE DE L'ALIMENT D'ALLAITEMENT}

La distribution à volonté de l'aliment d'allaitement chaud entraîne souvent des troubles digestifs, en particulier le météorisme de la caillette, maladie parfois mortelle habituellement chez les agneaux les plus gros et les plus vigoureux, et entraîner des pertes s'élevant jusqu'à $30 \%$. Ce trouble semble lié à une dilatation excessive de la caillette qui est causée par une trop grande consommation. Les agneaux nourris à volonté à l'aliment d'allaitement chaud tètent moins souvent, mais quand ils le font, ils absorbent une trop grande quantité de lait.

La nécessité de réduire les coûts en main-d'oeuvre tout en utilisant une méthode sûre d'allaitement à volonté a conduit à l'utilisation de l'aliment d'allaitement froid. L'aliment froid servi à volonté se conserve plus longtemps sans surir, élimine le météorisme de la caillette et produit une croissance des agneaux comparables à la croissance obtenue avec l'aliment chaud. Les agneaux nourris à l'aliment froid en absorbent de plus petites quantités à la fois, mais ils se nourrissent plus souvent, de sorte que leur consommation totale en $24 \mathrm{~h}$ est semblable à celle des agneaux nourris à l'aliment chaud. On recommande donc maintenant, de façon générale, que l'aliment d'allaitement soit servi froid, à environ $4^{\circ} \mathrm{C}$, en régime de distribution à volonté (voir la section «Systèmes de distribution»).

L'addition de formaline à l'aliment d'allaitement pour retarder la croissance bactérienne et le surissement est aussi recommandée pour l'élevage artificiel à l'échelle commerciale. Des études réalisées à la Station de recherches de Fredericton ont montré que l'incorporation de formaline, à une concentration inférieure à $0,10 \%$, à l'aliment d'allaitement chaud réduisait également de façon notable le météorisme de la caillette et n'avait pas d'effets négatifs sur la croissance de l'agneau, sur la valorisation des nutriments ou sur la muqueuse du système digestif.

$\mathrm{Au}$ Centre de recherches zootechniques, on a par la suite réalisé des expériences avec près de 2000 agneaux sur l'utilisation d'aliment d'allaitement chaud. La moitié des agneaux recevaient l'aliment froid et l'autre moitié l'aliment chaud. De la formaline, à raison de $0,10 \%$, était ajoutée à chacun des deux types d'aliments. Les deux traitements ont donné des agneaux en santé et vigoureux. Aucun cas de météorisme de la caillette ou d'un autre trouble digestif n'a été observé et le taux de survie était le même avec les deux types d'aliments. Les agneaux qui recevaient l'aliment chaud consommaient entre 1,2 et $1,4 \mathrm{~kg}$ de plus que les autres et étaient légèrement plus lourds au sevrage que ceux nourris à l'aliment froid. La réduction des coûts de main-d'oeuvre que le système d'alimentation à volonté permet de réaliser est donc sans danger pour la performance des agneaux, que l'aliment soit chaud ou froid.

Chaque producteur choisira la méthode d'alimentation qui lui convient en se fondant sur une évaluation du rapport coût-bénéfices. L'emploi de l'aliment d'allaitement chaud avec de la formaline permet 
d'utiliser un équipement plus simple et moins cher puisqu'on supprime ainsi les coûts de réfrigération. On peut ainsi réaliser des économies sur les coûts de main-d'oeuvre tout en utilisant le système de distribution à volonté sans aucun danger pour les agneaux. En revanche, l'utilisation de l'aliment d'allaitement chaud exige entre 1,2 et $1,4 \mathrm{~kg}$ de poudre de plus par agneau sevré. Le surcroît de gain obtenu par un taux d'ingestion plus fort peut compenser en partie le coût supplémentaire. Cependant, on ne peut compter sur cet avantage, car les méthodes ont démontré qu'une croissance compensatoire après le sevrage pouvait l'annuler. Si l'on utilise de la formaline, voir la section "Addition de formaline".

\section{SEVRAGE PRÉCOCE}

En raison du prix élevé de l'aliment d'allaitement, les agneaux doivent être sevrés aussitôt que possible, c'est-à-dire à l'âge de 3 semaines. Toutefois, il faut s'attendre à un certain arrêt de croissance et même à une légère perte de poids durant environ une semaine avant le retour à une croissance normale. Par après, le taux de gain des agneaux sevrés tôt est pratiquement identique à celui des agneaux sevrés plus tard.

L'arrêt de croissance est pratiquement éliminé si l'on retarde le sevrage d'une semaine, ce qui donne environ $1,5 \mathrm{~kg}$ de poids vif supplémentaire par agneau au moment de la mise au marché. Cependant, cette semaine de plus signifie $3 \mathrm{~kg}$ de poudre d'aliments d'allaitement de plus par agneau. Il est peu probable que les prix des aliments d'allaitement ou celui des agneaux varient suffisamment dans un avenir prévisible pour que le poids supplémentaire des agneaux puisse compenser le coût du supplément d'aliment d'allaitement additionnel. Il n'est donc pas justifiable économiquement de retarder le sevrage d'une semaine lorsque les agneaux ont de 3 à 4 semaines.

Une exception pourrait être faite pour les agneaux qui sont encore très petits à l'âge de 21 jours. L'expérience a démontré que les agneaux qui pèsent moins de $6 \mathrm{~kg}$ à 21 jours (habituellement moins de $4 \%$ de la population, et ce sont des agneaux qui sont très petits à la naissance) tireront profit d'une ou deux semaines supplémentaires à l'aliment d'allaitement.

Il est très important de s'assurer, au moment du sevrage, que tous les agneaux savent où se trouve l'eau. Il est possible que certains agneaux qui profitaient bien à l'alimentation liquide n'aient pas commencé à boire de l'eau. Si l'on n'y prend garde, ils pourraient mourir de déshydratation.

Les agneaux sevrés doivent recevoir un aliment à haute teneur énergétique afin de maximiser le gain quotidien de poids et la valorisation des aliments, de réduire les frais généraux et de raccourcir la période requise pour que les agneaux atteignent le poids du marché. La ration de croissance-finition devrait contenir environ 
$17 \%$ de protéines brutes jusqu'à l'âge de 100 jours et ensuite $14 \%$, jusqu'à ce qu'ils aient atteint le poids du marché et la qualité voulue.

La première journée du sevrage, on ne laisse aux agneaux que l'aliment complémentaire. On mélange ensuite des proportions croissantes de la ration de croissance-finition avec l'aliment complémentaire, jusqu'à ce que la mangeoire ne contienne que de la ration de croissance-finition, c'est-à-dire environ une semaine après le sevrage.

Le stress du sevrage sera réduit si les agneaux sont laissés dans l'enclos d'allaitement durant environ 2 semaines après le sevrage. On peut ensuite les placer dans des enclos plus grands ou des parquets d'engraissement.

\section{CHOIX DE L'ALIMENT D'ALLAITEMENT}

\section{SORTES}

La production des aliments d'allaitement requiert un équipement industriel spécialisé. Heureusement, plusieurs grandes provenderies offrent maintenant de bons aliments d'allaitement pour les agneaux, de même que des aliments d'allaitement de très bonne qualité pour les veaux, qui peuvent aussi être utilisés avec succès pour les agneaux. L'éleveur ne trouvera donc aucun avantage, économique ou autre, à préparer ses propres aliments d'allaitement en poudre. On se limitera donc ici à relever les points à considérer lors de l'achat des aliments d'allaitement.

Les meilleures performances s'obtiennent avec un aliment d'allaitement contenant au moins $24 \%$ de matières grasses et $24 \%$ de protéines, les protéines provenant exclusivement de poudre de lait de pulvérisation.

Le lait écrémé en poudre constitue la principale source de protéines dans la plupart des aliments commerciaux pour agneaux. Il est essentiel d'utiliser du lait écrémé en poudre de bonne qualité, séché par pulvérisation à basse température. Le traitement à haute température avant ou pendant le séchage nuit à la coagulation des protéines du lait dans l'estomac de l'agneau, ce qui abaisse la digestibilité de l'aliment pour les agnelets. On ne possède encore aucune donnée expérimentale sur les températures maximales de séchage. Il est donc conseillé de s'en tenir à la température indiquée pour la poudre de lait écrémé de consommation humaine.

Le babeurre en poudre constitue une excellente solution de rechange et il coûte souvent moins cher que le lait écrémé en poudre. Plusieurs compagnies remplacent des proportions variables de lait écrémé en poudre par du babeurre en poudre dans leurs préparations selon le rapport des prix des deux ingrédients. Dans des limites raisonnables, les poudres de lait écrémé et de babeurre semblent interchangeables, et aucun effet négatif ne semble résulter quand on 
fait varier les proportions de ces ingrédients dans les préparations d'aliments d'allaitement.

On préconisait autrefois une teneur en matières grasses de $30 \%$ et c'était devenu la pratique courante en Amérique du Nord. Cependant, les recherches ont démontré clairement que les aliments d'allaitement à $24 \%$ de matières grasses donnent lieu à une croissance aussi bonne, sinon meilleure, que les préparations à $30 \%$ de matières grasses. Cette réduction de la teneur en matières grasses abaisse d'environ $10 \%$ le prix de revient de l'aliment, car il est plus facile d'homogénéiser un aliment à faible teneur en gras. Les éleveurs ne devraient donc pas hésiter à utiliser des aliments d'allaitement à moindre teneur en matières grasses, lorsque ces aliments sont disponibles. La matière grasse peut être un mélange de suif, de saindoux, de matières grasses du lait ou d'huile de coco de qualité. Les autres huiles végétales ne sont pas à conseiller.

On a également de bons résultats avec les aliments d'allaitement dans lesquels une partie du lait écrémé en poudre est remplacée par de la caséine et du lactosérum ou du dextrose. Avec cette sorte d'aliment, la teneur en lactose (ou en sucres totaux) ne devrait pas dépasser 30 à $35 \%$, car on a noté que des teneurs en sucres supérieures à ces valeurs causaient des diarrhées. Cependant, lorsqu'on utilise la poudre de lait écrémé ou de babeurre de pulvérisation comme source de protéines, la teneur en lactose (ou en sucres totaux) se situe normalement entre 40 et $45 \%$. Aucun cas de diarrhée causé par un excès de sucre n'a été noté avec ces préparations.

Les agneaux peuvent également être élevés avec succès avec du lait de vache entier, si on peut disposer d'une source d'approvisionnement bon marché, ou avec de bons aliments d'allaitement pour veaux. Avec cette dernière sorte d'aliment, seuls doivent être considérés ceux qui contiennent au moins $20 \%$ de matières grasses et $20 \%$ de protéines provenant exclusivement de poudre de lait de pulvérisation. Les aliments d'allaitement pour veaux de qualité moindre, et donc moins coûteux, contiennent d'habitude moins de protéines ou de matières grasses, ou des deux, ou bien une partie des protéines peut provenir de produits végétaux. On évitera d'utiliser ces produits, car ils ne conviennent pas aux agneaux. Le gain de poids obtenu avec le lait de vache entier ou avec les aliments d'allaitement pour veaux sont d'environ $90 \%$ de ceux obtenus avec des aliments d'allaitement préparés spécialement pour les agneaux. L'utilisation de ces produits peut cependant ètre économique, car leur moindre coût compense largement la réduction, somme toute assez marginale de la croissance des agneaux.

Une autre possibilité est d'utiliser un mélange moitié de lait de vache et moitié d'aliment d'allaitement reconstitué pour agneaux. Avec ce mélange, la croissance des agneaux est identique à celle des agneaux qui sont nourris seulement avec l'aliment d'allaitement pour agneaux. 
Aux États-Unis, des doses d'antibiotiques relativement élevées (par exemple, de la néomycine au taux de $275 \mathrm{mg} / \mathrm{kg}$ ) ont été recommandées pour prévenir la diarrhée et les autres troubles digestifs. Des doses plus faibles (de la chlortétracycline au taux de 55 $\mathrm{mg} / \mathrm{kg}$ ) ont également été employées couramment dans des préparations expérimentales. Cependant, le Centre de recherches zootechniques n'a jamais utilisé d'antibiotiques pour l'élevage artificiel des agneaux. Il n'est pas nécessaire d'utiliser des doses non thérapeutiques d'antibiotiques dans les aliments d'allaitement pour agneaux et il n'est pas recommandé de le faire du moment qu'on observe de bonnes pratiques d'hygiène et de conduite des agneaux.

\section{PRÉPARATION}

Les aliments d'allaitement commerciaux doivent être reconstitués selon le mode d'emploi prescrit par le fabricant. Une partie (en poids) de la poudre d'aliment d'allaitement dans quatre parties d'eau donnent un produit dosant de 16 à $20 \%$ d'extrait sec, proportion habituellement recommandée. On ajoute d'abord environ la moitié de la quantité voulue d'eau chaude (environ 50 à $55^{\circ} \mathrm{C}$ ), pour que la poudre se dissolve complètement et que les globules de matières grasses demeurent homogénéisés. La température de l'eau ne doit pas dépasser $55^{\circ} \mathrm{C}$, car les protéines pourraient être endommagées. Une fois bien mélangée la poudre à l'eau chaude, ajouter la quantité d'eau (froide) qui reste et bien mélanger de nouveau. Réfrigérer le mélange avant de le servir aux agneaux si l'on utilise le système d'alimentation à froid, ou abaisser à la température du corps s'il doit être servi chaud.

\section{ADDITION DE FORMALINE}

L'addition de faibles concentrations de formaline à l'aliment d'allaitement comporte plusieurs avantages. La formaline retarde la croissance bactérienne et empêche l'aliment reconstitué de surir. De plus, elle prévient ou réduit de façon considérable le météorisme de la caillette chez les agneaux qui reçoivent l'aliment d'allaitement tiède. On recommande d'ajouter entre 0,05 et $0,10 \%$ de formaline à l'aliment reconstitué. Ces doses n'ont aucun effet nuisible sur les agneaux.

Si on emploie la formaline, on commence par mélanger la poudre avec l'eau tiède selon la méthode habituelle, puis on ajoute la formaline en mélangeant bien. Pour obtenir une concentration de $0,10 \%$, ajouter $1 \mathrm{~mL}$ (ou $1 \mathrm{~mL} / \mathrm{L}$ ) de formaline par kilogramme d'aliment liquide. Si l'on coupe de moitié la quantité de formaline utilisée, on obtiendra une concentration de $0,05 \%$. On utilise une seringue pour mesurer de façon précise la quantité de formaline. La concentration de formaline ne doit pas dépasser $0,10 \%$ sous peine de rendre l'aliment d'allaitement inappétent pour les agneaux, qui consommeraient moins et auraient donc une croissance plus lente. 
La formaline est une solution qui contient $35 \%$ (en poids) de formaldéhyde. Comme c'est un produit chimique dangereux, on doit le manipuler avec soin pour éviter les blessures. Les vapeurs qui s'en dégagent peuvent irriter les yeux et le nez. Si la formaline vient en contact avec la peau, laver avec soin l'endroit atteint; si on en reçoit dans les yeux, les bassiner pendant 15 min à l'eau courante, puis appeler un médecin. Moyennant les précautions nécessaires, la formaline n'est pas plus dangereuse que tout autre produit chimique d'usage courant à la ferme.

\section{SYSTÈMES DE DISTRIBUTION}

\section{PROTECTION DES TÉTINES}

Les agneaux peuvent endommager considérablement les tétines en les mordillant, particulièrement quand ils sont plus âgés. La plupart du temps, ces dommages surviennent lorsque les agneaux saisissent les tétines par le côté avec leurs molaires. On peut perdre des quantités importantes de lait si l'on ne protège pas les tétines. Dans un système de distribution à volonté, il faut entourer les tétines de protecteurs en métal ou en plastique pour éviter que les agneaux ne les percent sur le côté (fig. 5 à 8). Les protecteurs en plastique fabriqués à partir de bouteilles vides, soigneusement nettoyées, sont également efficaces. Les protecteurs peuvent aussi contribuer à guider les agneaux vers les tétines lorsque ceux-ci apprennent à têter. On peut aussi protéger les tétines en les plaçant au fond de trous de 8 à $10 \mathrm{~cm}$ de diamètre (ou encore mieux, $6 \mathrm{~cm}$ de largeur sur $10 \mathrm{~cm}$ de hauteur) pratiqués dans une planche de contre-plaqué, de telle sorte que l'agneau ne puisse avoir accès à la tétine qu'en introduisant son museau dans le trou (fig. 1). Il semble que l'on puisse également protéger les tétines en les montant sur une plaque en métal ou en bois munie d'une cloison de chaque côté des tétines, en laissant de 8 à $10 \mathrm{~cm}$ entre la tétine et la cloison.

\section{ALIMENTATION AU SEAU À TÉTINES}

On peut facilement installer une ou plusieurs tétines sur un seau ou un autre contenant en plastique. On pratique un ou plusieurs trous, selon le cas, au bas du seau et on le suspend à la cloison de l'enclos, de façon à ce que les agneaux puissent se servir eux-mêmes (fig. 3). Les principaux avantages de ce système simple sont le coût modique et la facilité de nettoyage. Le contenu du récipient peut être gardé froid en y laissant flotter une bouteille en plastique remplie d'eau glacée ou un sachet réfrigérant commercial. Les principaux inconvénients du système tiennent à la difficulté d'empêcher les agneaux de mordiller les tétines. Une tétine abîmée peut entraîner la perte de tout le contenu du récipient. 


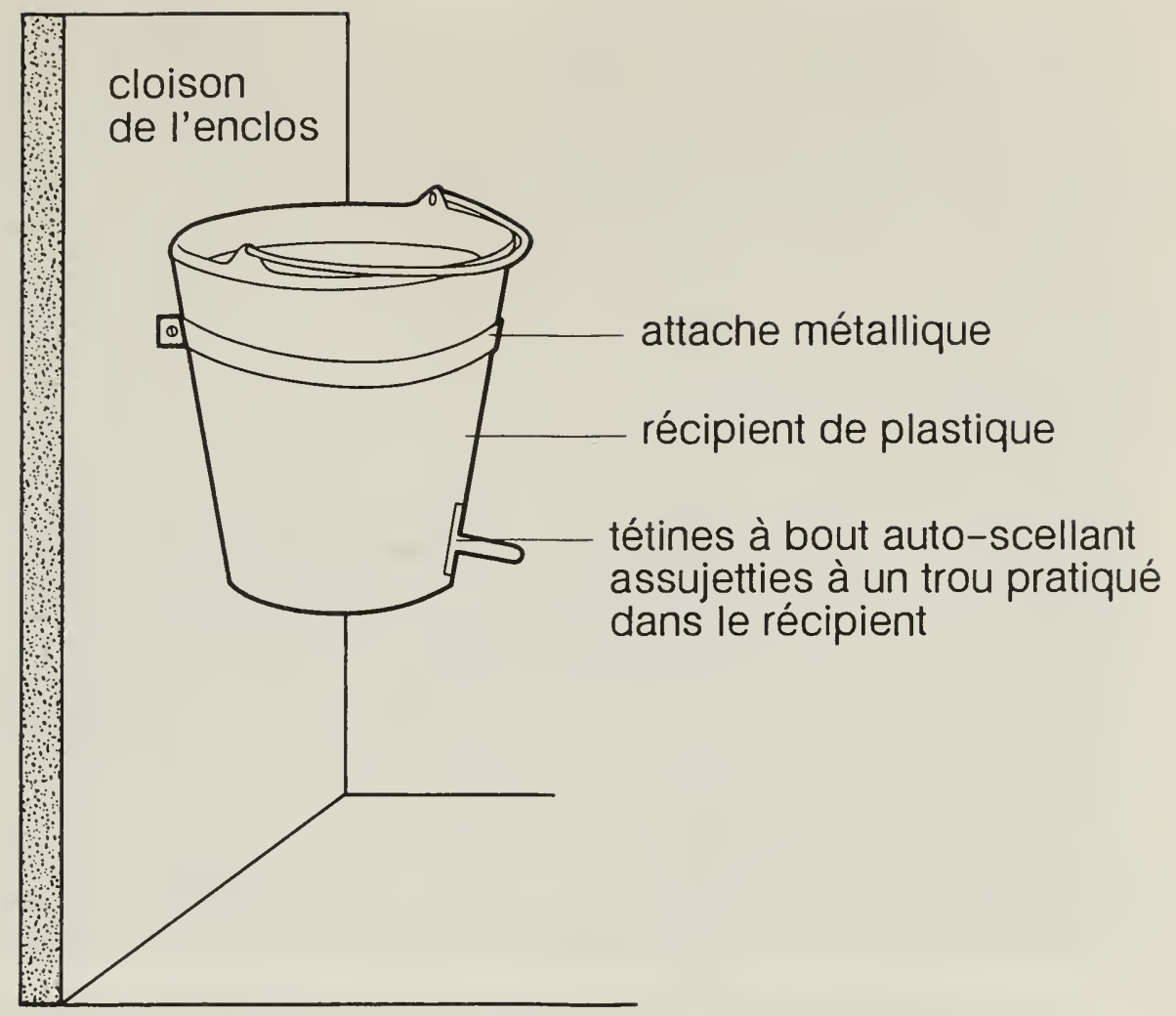

Fig. 3 Seau peu coûteux utilisé pour l'alimentation de petits groupes d'agneaux (d'après Spedding et al., 1976).

\section{NOURRISSEURS À PLUSIEURS TÉTINES}

On peut fixer les tétines à un raccord en métal ou en plastique fixé à un trou pratiqué dans la cloison de l'enclos, puis les relier par un tube en plastique au récipient qui contient l'aliment d'allaitement (fig. 4). Un même récipient peut alors approvisionner plusieurs tétines. On peut éviter les pertes d'aliments causés par l'endommagement des tétines en plaçant le contenant à un niveau inférieur à celui de la tétine. Le principal inconvénient de ce système est qu'il est plus difficile d'apprendre aux agneaux à boire, car ils doivent sucer plus longtemps et plus fort pour que l'aliment remonte dans le tube et atteigne la tétine. Beaucoup de petits agneaux peu vigoureux n'y arrivent pas. L'installation d'un clapet de pied pour éviter que l'aliment d'allaitement ne retombe dans le récipient facilite l'opération, mais il peut être difficile de maintenir le clapet en bon état de fonctionnement. On peut fabriquer un clapet de pied en plaçant un roulement à billes sur le dessus de la cartouche d'un stylo à bille, ellemême fixée à l'extrémité du tube d'alimentation. On peut aussi se procurer des clapets commerciaux dans les magasins de pièces d'automobile (clapets pour l'essuie-glaces) ainsi que dans les quincailleries et autres magasins d'appareils ménagers. 


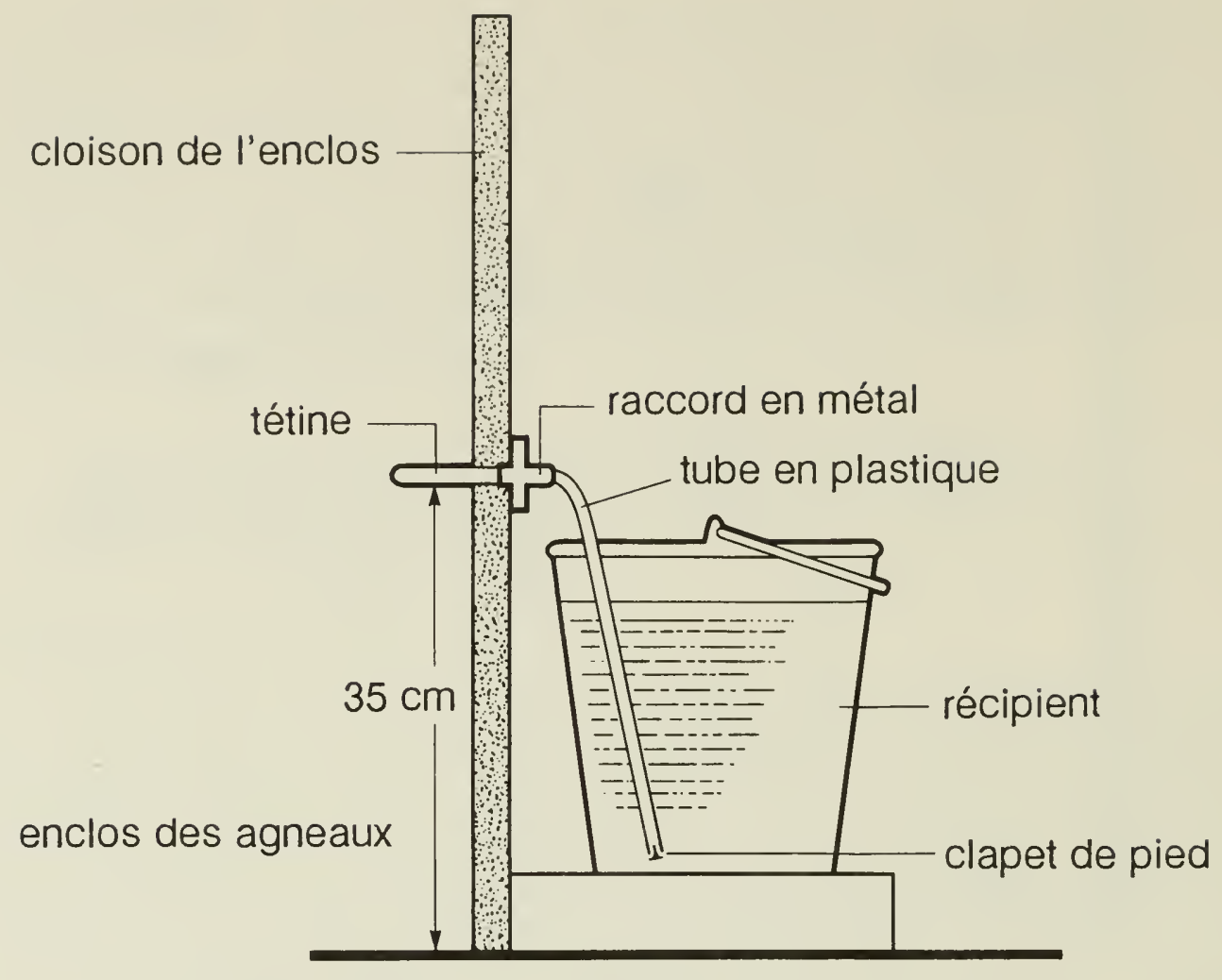

Fig. 4 Appareil simple pour l'alimentation de groupes d'agneaux. Si la tétine est endommagée, l'aliment d'allaitement ne coule pas, comme ce serait le cas avec le système illustré à la figure 3 (d'après Spedding et al., 1976).

Il existe sur le marché un système dont les tétines sont fixées à la partie inférieure du récipient, de façon à ce que l'aliment coule par gravité et que plusieurs agneaux se nourrissent en même temps. Pour garder le lait froid, on peut placer dans le seau des sacs en plastique ou des bouteilles contenant de la glace.

\section{RAMPES NOURRICIÈRES}

Les rampes nourricières comme celles illustrées aux figures 5 à 8 sont relativement faciles à confectionner et peu coûteuses. Elles peuvent être montées à partir d'articles de plomberie faciles à trouver. Une rampe nourricière se compose de tétines reliées à des tuyaux ou à des canalisations, dans lesquels l'aliment d'allaitement s'écoule, par gravité ou à l'aide d'une pompe, à partir d'une source d'approvisionnement. L'aliment peut être réfrigéré, chauffé ou laissé à la température de la pièce.

Les tétines utilisées pour la rampe doivent être conçues ou modifiées de manière à ce qu'elles ne coulent pas lorsqu'elles sont remplies de l'aliment d'allaitement. Les tétines Lamblux ${ }^{\circledR}$ (fig. 5) ainsi que les tétines pour biberons (fig. 7), sur lesquelles une petite fente verticale a été pratiquée à l'aide d'une lame tranchante ont été utilisées avec succès sur les rampes nourricières. 

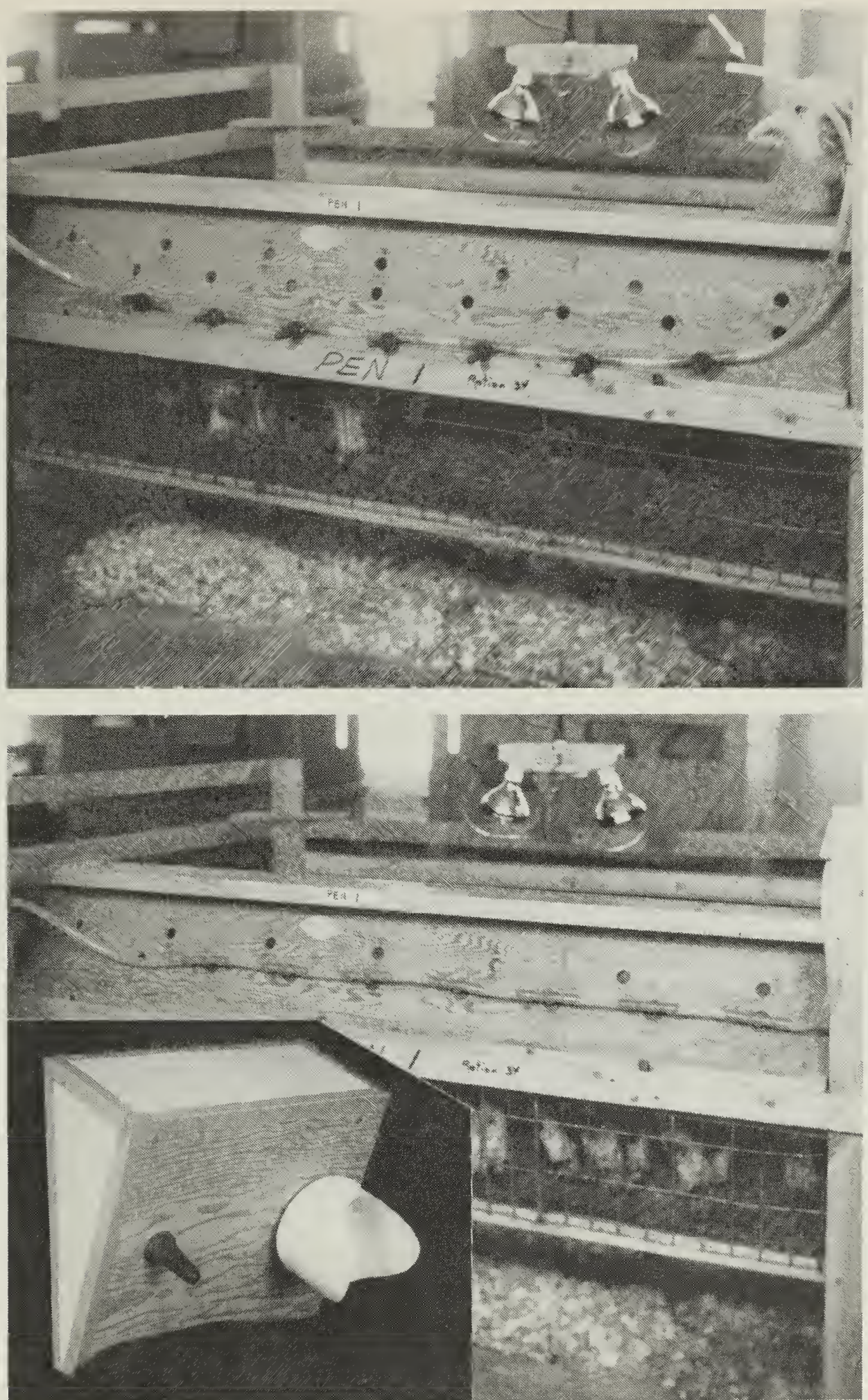

Fig. 5 (a) Rampe nourricière pour l'alimentation d'un petit groupe d'agneaux et tétines Lamblux ${ }^{\circledR}$, non encore installées dans les orifices d'alimentation. Les tubes en plastique ont un diamètre de $1,25 \mathrm{~cm}$; à droite, l'extrémité du tube (flèche) a été laissée ouverte pour éviter qu'il ne se crée une pression à l'intérieur du système. (b) Rampe nourricière sur laquelle les tétines ont été introduites dans les orifices de $2,5 \mathrm{~cm}$ de diamètre pratiquées dans une planche de contreplaqué. (en médaillon) Tétines qui sortent de la planche inclinée vers le bas, avec et sans protecteur en plastique. Le protecteur a été fabriqué avec une bouteille de détergent de $1 \mathrm{~L}$. 


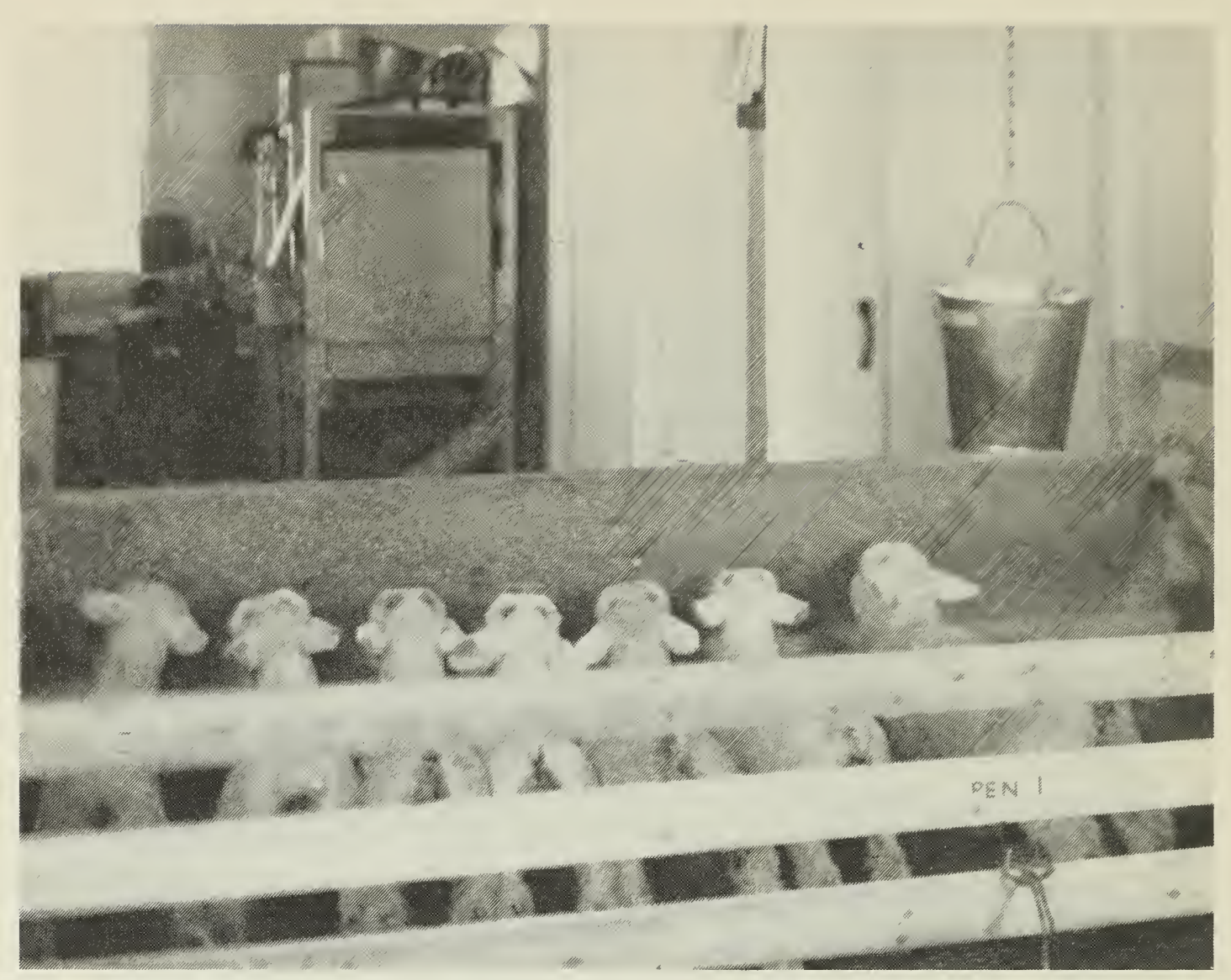

Fig. 6 Agneaux qui tètent l'aliment d'allaitement chaud à l'aide de la rampe nourricière illustrées aux figures $5 a$ et $b$.

À la Station de recherches de Fredericton (fig. 5 et 6 ), une rampe nourricière peu coûteuse a été fabriquée à partir d'un tube en plastique souple de $1,25 \mathrm{~cm}$. Des raccords en $\mathrm{T}$ de $1,25 \mathrm{~cm}$ en plastique rigide ont été introduits à tous les $20 \mathrm{~cm}$. La partie du $\mathrm{T}$ à laquelle la tétine est reliée est coupée de façon à n'avoir plus qu'une ou deux stries, pour éviter que le plastique dur ne glisse trop loin dans la tétine. L'autre extrémité du tube est laissée ouverte, comme l'illustre la figure $5 a$, pour éviter qu'il ne se crée une pression à l'intérieur de la canalisation et qu'il ne se produise des fuites aux tétines non utilisées lorsqu'un agneau suce une autre tétine. Il faut un minimum de six tétines par rampe nourricière pour ne pas que les tétines libres coulent durant la têtée. La planche sur laquelle les tétines sont fixées (fig. $5 b$ ) est placée à un angle d'environ $65^{\circ}$ pour imiter la forme du pis de la brebis. Les tétines peuvent être espacées de plus de $20 \mathrm{~cm}$, selon la forme de l'enclos et le nombre d'agneaux à nourrir, mais il faut laisser au moins $20 \mathrm{~cm}$, sinon les agneaux seront entassés.

Des canalisations rigides ont également été utilisées avec succès, mais elles sont plus difficiles à nettoyer. Les figures 7 et 8 illustrent des rampes nourricières fabriquées de cette façon. L'aliment d'allaitement peut être gardé dans un seau ouvert à la température de la pièce ou être réfrigéré (fig. 9). Un réfrigérateur usagé ou un petit 
réfrigérateur à lait utilisé dans les restaurants peut contenir suffisamment d'aliment pour nourrir jusqu'à 50 agneaux. On peut pratiquer un trou sur le côté du réfrigérateur afin d'y introduire la canalisation qui relie la rampe nourrice au contenant de l'aliment, comme l'indique la figure 9.

a

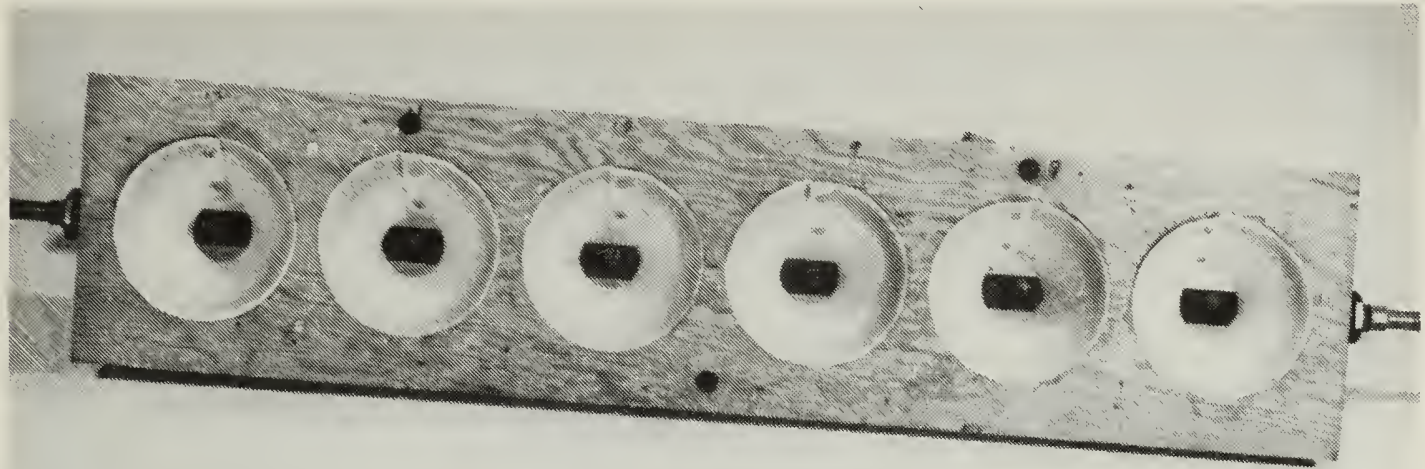

b

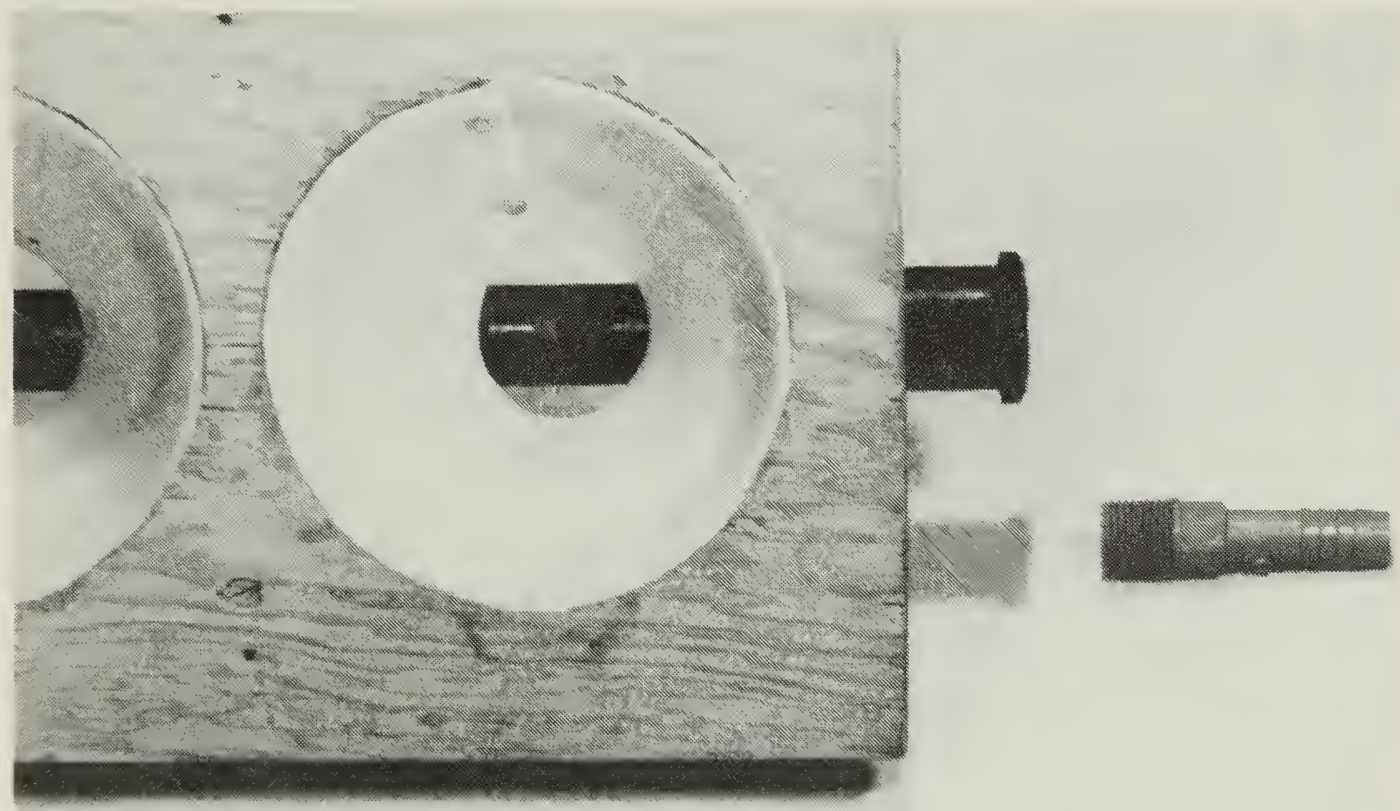

Fig. 7 (a) Autre type de rampe nourricière; les tétines doivent être espacées d'au moins $20 \mathrm{~cm}$. (b) Éléments d'une unité d'alimentation de la rampe : protecteur en plastique, tube en plastique perforé et raccord de réduction, bonde en plastique et tétine de biberon. 


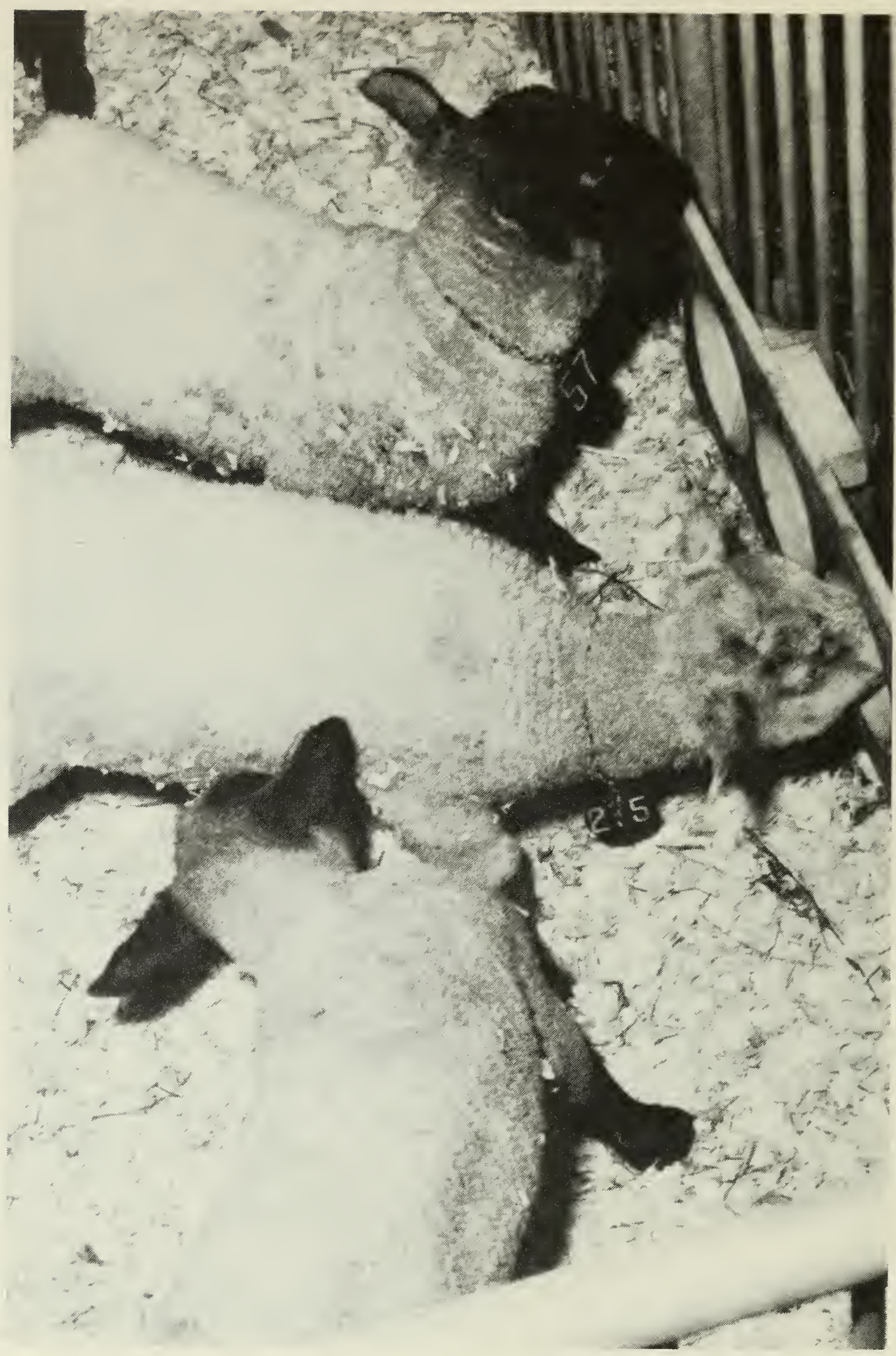

Fig. 8 Agneaux qui tètent l'aliment d'allaitement froid circulant dans une rampe nourricière semblable à celle présentée aux figures $7 a$ et $b$. 
La circulation continue de l'aliment d'allaitement entre la rampe nourricière et le réfrigérateur maintient l'aliment à une température froide constante. Une petite pompe à pression positive en plastique (fig. 9), peu coûteuse, entraînée par un moteur à vitesse variable fonctionnant à environ $100 \mathrm{r} / \mathrm{min}$ a donné de bons résultats à la Station de recherches de Fredericton. On a également utilisé de petites pompes à engrenages qui fonctionnent à faible vitesse. Une petite pompe centrifuge s'est révélée inefficace, car elle produisait beaucoup de mousse et entraînait la séparation des matières grasses dans l'aliment d'allaitement.

Il est recommandé de prévoir un système d'arrêt automatique de la pompe lorsque l'aliment vient à manquer. Le système de circulation de l'aliment doit être conçu de manière à n'exercer qu'une très faible pression sur les tétines, sinon celles-ci couleront. Dans le système illustré à la figure 9, l'aliment d'allaitement coule par gravité du contenant réfrigéré à la rampe nourricière puis est retourné dans le contenant par la pompe.

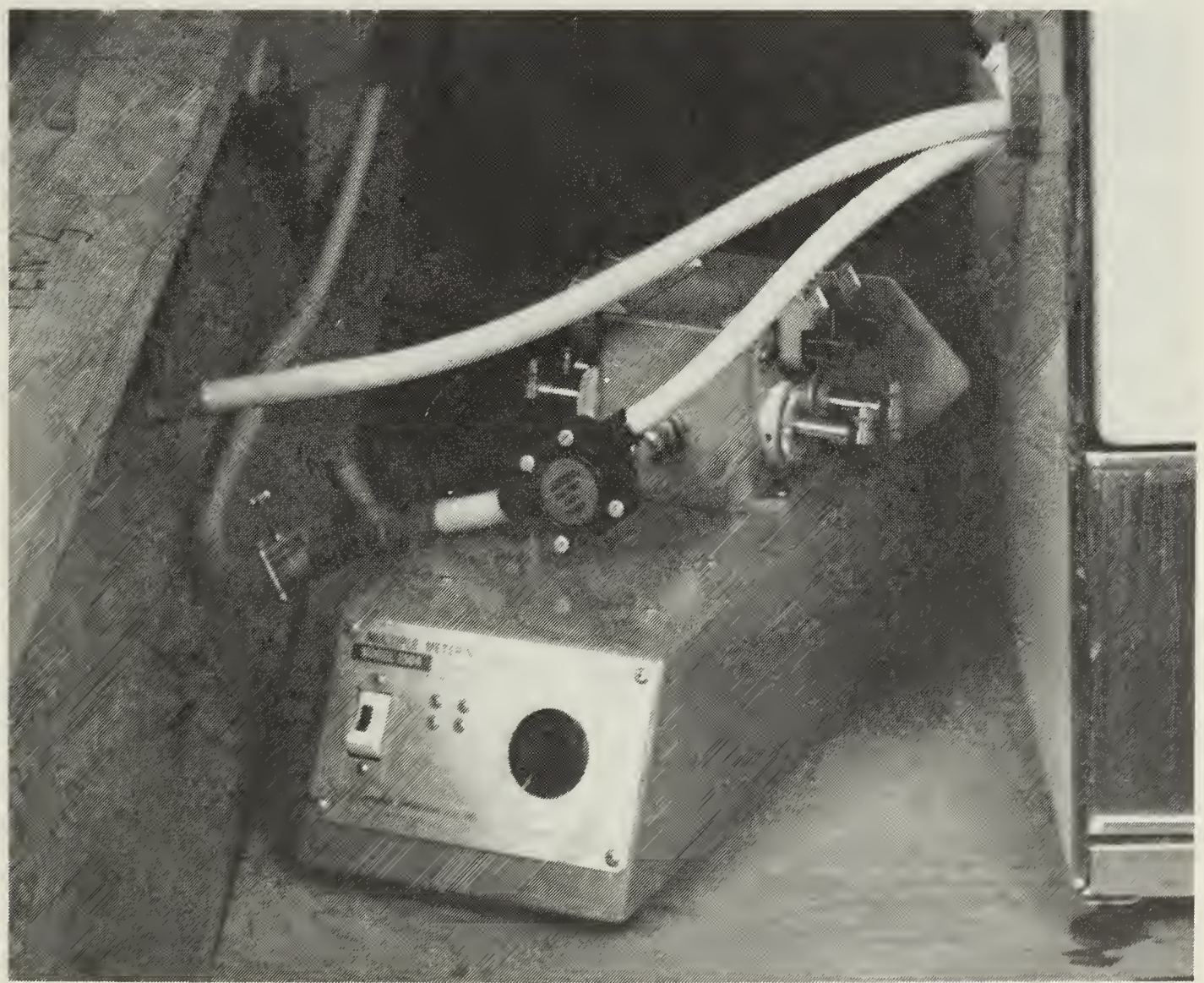

Fig. 9 Pompe en plastique reliée à un moteur à vitesse variable. L'aliment d'allaitement coule par gravité du contenant placé à l'intérieur du réfrigérateur vers les tétines, puis retourne dans le contenant grâce à la pression exercée par la pompe. 


\section{HYGIÈNE}

Pour obtenir une croissance optimale des agneaux, il est essentiel de nettoyer régulièrement tout le matériel de distribution de l'aliment d'allaitement et d'appliquer de bonnes mesures d'hygiène. L'aliment d'allaitement liquide est un milieu très propre à la croissance de bactéries et il surit vite s'il est laissé à la température de la pièce. Il est souhaitable de nettoyer chaque jour les tétines, les canalisations ou les tuyaux. Pour nettoyer et désinfecter les ustensiles, les tétines ou autres appareils, les rincer tour à tour à l'eau froide, à l'eau chaude additionnée de détergent, à l'aide d'une solution d'hypochlorite ou de tout autre désinfectant. On peut devoir recourir à la brosse pour enlever les matières grasses lors du lavage à l'eau chaude. Terminer le nettoyage par un bon rinçage à l'eau froide. Il ne doit rester aucune trace de détergent. Les réservoirs d'aliment d'allaitement doivent être nettoyés deux ou trois fois par semaine, ou plus souvent si l'on constate que l'aliment surit plus vite. La fréquence de nettoyage est déterminée par le type de contenant utilisé, la température du lait et l'addition de formaline, qui empêche le lait de surir. Les systèmes de canalisation complexes peuvent être nettoyés par recirculation, à intervalles déterminés.

Dans les systèmes d'alimentation à volonté, l'aliment doit être disponible en tout temps; le temps nécessaire au nettoyage et à la désinfection du matériel doit donc être très court. C'est pourquoi il est utile d'avoir en double tous les éléments du système d'alimentation utilisé (rampes nourricières, tétines et autres). On peut ainsi utiliser le matériel propre pendant que l'on procède au nettoyage de l'autre. De cette façon, les agneaux ne sont jamais privés d'aliments et le lavage peut se faire lorsque le temps le permet.

\section{MALADIES ET TROUBLES DIGESTIFS}

Une injection de vitamines $\mathrm{A}, \mathrm{D}$ et $\mathrm{E}$ à la naissance aide à prévenir les maladies et les infections. Si la dystrophie musculaire, ou maladie du raide, sévit dans la région, on administrera aux agneaux une injection de vitamine $\mathrm{E}$ et de sélénium à la naissance, puis une seconde injection au moment du sevrage. Le sélénium peut aussi être ajouté par le fabricant à l'aliment en poudre. Le supplément de sélénium ne doit pas être administré sous plus d'une forme, c'est-àdire que si l'aliment d'allaitement en contient déjà, éviter d'en injecter aux agneaux. Il est à conseiller aussi de fournir du sélénium aux brebis, soit dans leur ration de céréales soit dans un supplément minéral servi à volonté.

Les agneaux recevant l'aliment d'allaitement à volonté puis des aliments solides à haute valeur énergétique sont sujets, à l'âge de 3 ou 4 semaines, à la maladie de la suralimentation (rein pulpeux ou 
entérotoxémie). Ces agneaux doivent donc être vaccinés, à l'âge de 7 à 10 jours, avec une préparation multiple de clostridium ou une préparation du type $\mathrm{D}$ (utiliser la moitié de la dose recommandée pour les agneaux d'engraissement). On donne une vaccination de rappel deux semaines plus tard. Ou encore, on peut vacciner la brebis environ 2 à 4 semaines avant l'agnelage et les anticorps qu'elle produira seront alors transmis à l'agneau par le colostrum. Dans ce cas, il faudra donner aux agneaux une vaccination de rappel à l'âge de 49 à 56 jours.

Le météorisme de la caillette peut constituer un problème chez les agneaux élevés au lait de remplacement. Les gaz qui causent le météorisme de la caillette sont produits par un microorganisme, le Lactobacillus fermentum. Les faibles concentrations d'antibiotiques parfois utilisées dans les aliments d'allaitement pour agneaux n'ont aucun effet sur cet organisme; cependant, sa croissance peut être fortement ralentie par les faibles concentrations de formaldéhyde $(0,05$ à $0,10 \%$ de formaline $)$ recommandées pour empêcher le sûrissement (voir la section "Addition de formaline»).

En général, les agneaux recevant un aliment d'allaitement de bonne qualité ne souffrent pas de diarrhée. Cependant, ce problème peut survenir si l'on utilise un aliment à faible teneur en matières grasses et à forte teneur en lactose, ou encore à forte teneur en amidon ou en graisses polyinsaturées.

\section{PERFORMANCE DES AGNEAUX NOURRIS AUX ALIMENTS D'ALLAITEMENT}

Dans de bonnes conditions de gestion et d'alimentation, la croissance des agneaux nourris aux aliments d'allaitement s'est avérée égale ou supérieure à celle des agneaux allaités par la mère. Jusqu'à l'âge de 3 à 4 semaines, la croissance des agneaux nourris à l'aliment d'allaitement est comparable à celle d'agneaux uniques semblables allaités par la brebis, et en général meilleure que celle de jumeaux allaités par la mère. L'agneau devrait engraiser de 0,25 à $0,30 \mathrm{~kg}$ par jour pendant toute la période où il est nourri avec l'aliment d'allaitement. La concurrence pour le lait étant éliminée avec l'élevage artificiel, la croissance des agneaux issus de portées de tailles différentes (uniques, jumeaux, triplés, etc.) sera à peu près la même. Lorsqu'une fois sevrés, les agneaux reçoivent des aliments à haute teneur énergétique (concentrés), le gain de poids devrait demeurer à peu près le même jusqu'à l'âge d'environ 100 jours.

Les agneaux en croissance rapide consomment chaque jour jusqu'à $0,5 \mathrm{~kg}$ de poudre d'aliment d'allaitement de bonne qualité. C'est un aliment qu'ils valorisent très bien et il ne leur en faut qu'environ $1 \mathrm{~kg}$ en poudre pour chaque kilogramme de gain de poids.

La quantité totale d'aliment d'allaitement nécessaire pour nourrir un agneau varie selon le système de distribution, la composition de 
l'aliment, l'âge de l'animal au sevrage et le poids de l'agneau à la naissance. Les agneaux sevrés et nourris à volonté à l'aliment d'allaitement pendant 3 ou 4 semaines consomment en général de 7 à $9 \mathrm{~kg}$ de poudre.

Le taux de mortalité chez les agneaux nourris à l'aliment d'allaitement est en général inférieur à $5 \%$. Les mortalités sont moins fréquentes chez les agneaux élevés artificiellement que chez les agneaux nourris par la mère, car l'élevage artificiel comporte moins de risques d'accidents ou d'insuffisance d'alimentation.

\section{LECTURES CONSEILLÉES}

Ainsworth, L.; Heaney, D.P.; Fiser, P.S.; Langford, G.A.; Shrestha, J.N.B.; Leger, D.A. 1987. Accroissement de la capacité de rendement des systèmes de production d'agneaux - Recherche et technologie. Agric. Can. Bull. Tech. 1987-11F. 91 p. (Pour obtenir un exemplaire gratuit, s'adresser au directeur du Centre de recherches zootechniques, Direction générale de la recherche, Agriculture Canada, Ottawa (Ont.) K1A 0C6).

Frederickson, K.R.; Jordan, R.M.; Terrill, C.E. 1980. Rearing lambs on milk-replacer diets. U.S. Dept. Agric. Farmers' Bull. No 2270.

Heaney, D.P.; Shrestha, J.N.B.; Peters, H.F. 1983-1984. Reducing the cost of milk replacer for the artificial rearing of lambs. Sheep Canada 8(4):7.

Heaney, D.P.; Shrestha, J.N.B. 1984. Management tips for reducing artificial rearing costs for lambs. Sheep Canada 9(4):3-4.

Hess, E.W. 1971. The milk-bar "foot-valve». The Shepherd 16(3):10.

Sheep Industry Development Program, Inc. 1975. The Sheepman's Production Handbook, 2e éd. Abegg Printing, Denver (Colorado).

Spedding, C.R.W., et al. 1976. Artificial rearing of lambs. Technical Report, Sheep Improvement Service, Meat and Livestock Commission, U.K., 35 p. 
TABLE DE CONVERSIONS

Facteur

Unités impériales

de conversion

Unités mètriques

Longueur

$\begin{array}{lrr}\text { pouce } & \times 25 & \text { millimètre }(\mathrm{mm}) \\ \text { pied } & \times 30 & \text { centimètre }(\mathrm{cm}) \\ \text { verge } & \times 0.9 & \text { mètre }(\mathrm{m}) \\ \text { mille } & \times 1.6 & \text { kilomètre }(\mathrm{km})\end{array}$

Surtace

$\begin{array}{ll}\text { pouce carré }\left(\mathrm{go}^{2}\right) & \times 6,5 \\ \text { pied carré }\left(p i^{2}\right) & \times 0,09 \\ \text { verge carrée }\left(\mathrm{v}^{2}\right) & \times 0,836 \\ \text { mille carré } & \times 259 \\ \text { acre } & \times 0,40\end{array}$

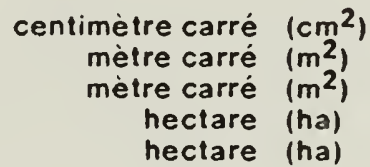

Volume

$\begin{array}{ll}\text { pouce cube } & \times 16 \\ \text { pied cube } & \times 28 \\ \text { verge cube } & \times 0,8 \\ \text { once liquide } & \times 28 \\ \text { chopine } & \times 0,57 \\ \text { pinte } & \times 1,1 \\ \text { gallon (R.-U.) } & \times 4,5 \\ \text { gallon (E. -U.) } & \times 3,8\end{array}$

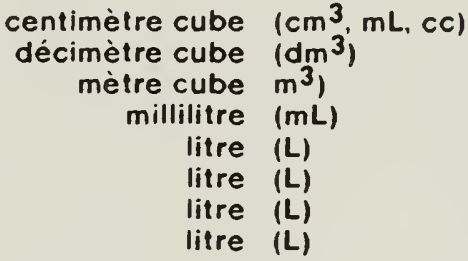

Masse

$\begin{array}{ll}\text { once } & \times 28 \\ \text { livre } & \times 0,45 \\ \text { tonne (courte) } & \times 0.9\end{array}$

gramme (g) kilogramme (kg) tonne (t)

\section{Température}

degrés Fahrenheit

$$
\begin{aligned}
& \left({ }^{\circ} \mathrm{F}-32\right) \times 0,56 \\
& \text { ou }\left({ }^{\circ} \mathrm{F}-32\right) \times 5 / 9
\end{aligned}
$$

degrés

Celsius $\left({ }^{\circ} \mathrm{C}\right)$

\section{Pression}

livres par pouce carré $\times 6,9$

kilopascal (kPa)

Puissance

$\begin{aligned} \text { cheval-vapeur } & \times 746 & \text { watt } & (\mathrm{W}) \\ & \times 0,75 & \text { kilowatt } & (\mathrm{kW})\end{aligned}$

\section{Vitesse}

pieds par seconde $\quad \times 0,30 \quad$ mètres par seconde $(\mathrm{m} / \mathrm{s})$

milles par heure $(\mathrm{mph}) \times 1,6 \quad$ kilomètres par heure $(\mathrm{km} / \mathrm{h})$

\begin{tabular}{|c|c|c|c|}
\hline gallons par acre & $\times 11,23$ & litres par hectare & (Lha) \\
\hline pintes par acre & $\times 2,8$ & litres par hectare & (Lha) \\
\hline chopines par acre & $\times 1.4$ & litres par hectare & (Lha) \\
\hline onces liquides par acre & $\times 70$ & millilitres par hectare & (mLha) \\
\hline tonnes par acre & $\times 2,24$ & tonnes par hectare & (t/ha) \\
\hline livres par acre & $\times 1,12$ & $\begin{array}{r}\text { kilogrammes par } \\
\text { hectare }\end{array}$ & (kgha) \\
\hline $\begin{array}{l}\text { onces par acre } \\
\text { plants par acre }\end{array}$ & $\begin{array}{l}\times 70 \\
\times \quad 2,47\end{array}$ & $\begin{array}{l}\text { grammes par hectare } \\
\text { plants par hectare }\end{array}$ & $\begin{array}{l}\text { (gha) } \\
\text { (plants/ha) }\end{array}$ \\
\hline
\end{tabular}

\section{Agriculture}


